\title{
Measurement of the remnant magnetic-field in Lorentz mode using permalloy
}

\author{
Haihua $L I U^{1)}$, Xiaokun DUAN ${ }^{2)}$, Renchao CHE ${ }^{1)}$, Zhifeng $W A N G^{1)}$ \\ and Xiaofeng $D U A N^{1) *}$ \\ 1) Beijing Laboratory of Electron Microscopy, Beijing National Laboratory for Condensed Matter Physics, \\ Institute of Physics, Chinese Academy of Sciences, Beijing 100190, China \\ 2) Advanced Technology \& Materials Co., Ltd., Beijing 100081, China \\ Manuscript received 21 January 2009; in revised form 1 May 2009
}

\begin{abstract}
A novel method was reported to measure the remnant magnetic field in Lorentz mode in a FEI Tecnai F20 transmission electron microscope equipped with a Lorentz lens. The movement of the circle Bloch line of the cross-tie wall in Permalloy is used to measure the remnant magnetic field by tilting the specimen and adjusting the objective lens current. The remnant magnetic field is estimated to be about $17 \mathrm{Oe}$, in a direction opposite to that of the objective lens magnetic field. The remnant magnetic field can be compensated by adjusting the value of the objective lens current.

KEY WORDS Lorentz electron microscopy; Lorentz lens; Remnant magnetic field; Permalloy; Cross-tie walls
\end{abstract}

\section{Introduction}

Transmission electron microscopy (TEM) is a powerful tool for high-resolution studies of magnetic microstructures such as domain wall arrangements, magnetization distribution and magnetic phase transformations by means of Lorentz electron microscopy and electron holography ${ }^{[1-4]}$. Magnetic microstructure observations should however be carried out using an objective lens that can be switched off and/or a magnetically shielded objective lens (a so-called Lorentz lens) in order to avoid the loss of the original domain structure due to interaction with the strong magnetic field produced by a conventional objective lens ${ }^{[5]}$. The magnetic field in the specimen position can be decreased to zero by using a Lorentz lens installed in a TEM. However, in most cases, a small remnant magnetic field still remains, even though the objective lens is already switched off or shielded, and this remnant field may be too strong to allow observation for magnetic domains in soft magnetic materials. Measurement of the remnant magnetic field is essential for Lorentz TEM investigations. Various methods have been used to characterize the remnant magnetic field in the specimen position in a $\mathrm{TEM}^{[6]}$. One option is to use a specialized Hall probe holder. This method can give very accurate measurements, but is a very expensive solution. Lau et al. reported

\footnotetext{
*Corresponding author. Professor; Tel.: +86 10 82648008; Fax: +86 1062561422.

E-mail address: xfduan@blem.ac.cn (Xiaofeng DUAN)
}

DOI: $10.1016 / \mathrm{S} 1006-7191(08) 60120-9$ 
measurement of the remnant magnetic field using a TEM holder adapted to include a commercially available Hall probe. However, this method leads to large uncertainties in the measurement due to the difficult of positioning the probe accurately in the specimen position ${ }^{[6]}$. Another method is to utilize a test sample with known magnetic response under an applied magnetic field ${ }^{[7]}$.

A novel method was reported to measure the remnant magnetic field at the exact specimen position through investigating the movement of a circle Bloch line along the main wall of the cross-tie wall by tilting the specimen of a soft magnetic material.

The material chosen for this method is 80 at. pct Ni Permalloy. This material has good magnetic properties under a weak magnetic field, such as a very high initial magnetic permeability $\left(\mu_{i}\right)$ and a maximal magnetic permeability $\left(\mu_{\mathrm{m}}\right)$, a higher magnetic saturation induction $\left(B_{\mathrm{s}}\right)$ and a low coercive field $\left(H_{\mathrm{c}}\right)^{[8,9]}$. Cross-tie walls in Permalloy thin film were first observed by Huber using bitter pattern methods in $1958^{[10]}$. The magnetic flux closure within the plane of the film as studied by Huber, is shown in Fig.1a. The main wall is cut at regular intervals by short, perpendicular "cross ties", each of which terminates in a free single end. It is well established that domain wall creeping of the cross-tie walls is connected with circle Bloch line movements and a variation of the main wall curvature ${ }^{[11-13]}$. Fig.1b shows that circle Bloch line moves along the main wall between pairs of adjacent cross-ties under the influence of a hard-axis field normal to the main wall. Fig.1c shows the main wall bends by buckling of located parts of the wall under the influence of an easy-axis field parallel to the main wall. The displacement of the circle Bloch lines in the cross-tie walls can be considered as being linearly related to the hard-axis direction field for small displacements ${ }^{[11-13]}$.

\section{Experimental}

A TEM Permalloy specimen was prepared by mechanical thinning, followed by ion-milling in an argon atmosphere to electron transparency using a Gatan ion milling system (model $600 \mathrm{~B}$ ) with a liquid nitrogen

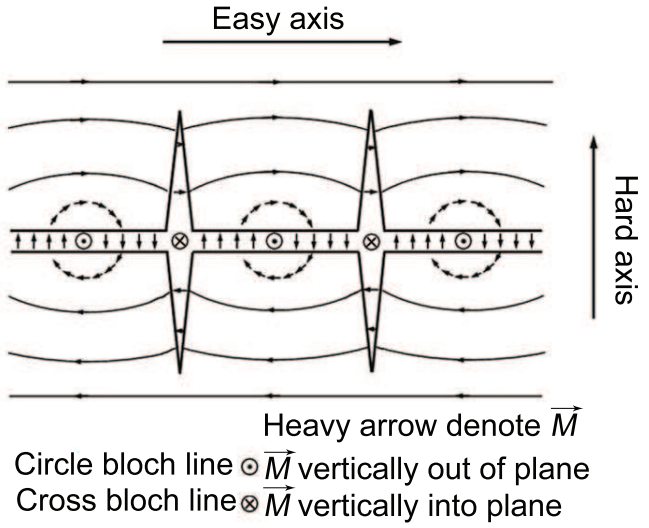

(a)

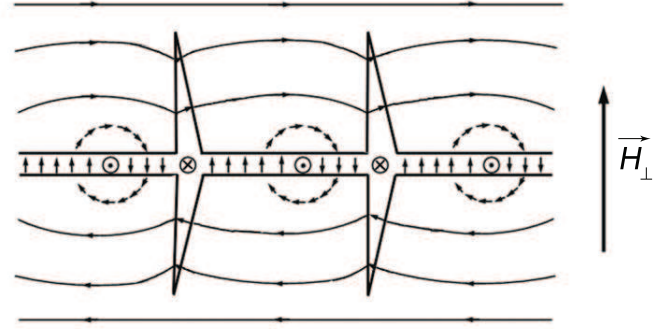

(b)

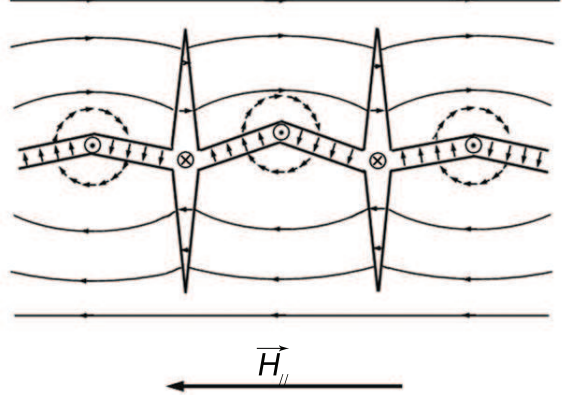

(c)

Fig.1 (a) Diagram of the cross-tie wall structure in a Permalloy thin film showing flux closures within the plane of the film, (b) changes under the influence of a transverse field in the hard axis direction, (c) changes under the influence of a transverse field in an easy axis direction field. 
cold-stage. The in situ Lorentz electron microscopy measurements of remnant magnetic field were carried out using a FEI Tecnai F20 field emission gun (FEG) TEM equipped with a Lorentz lens. In order to avoid magnetic saturation of the magnetic specimen, the objective lens was be switched off by setting the TEM into Lorentz mode before the specimen holder was put into the TEM column. By tilting the specimen, as seen in Fig.2, the resulting in-plane component of the remnant magnetic field causes a movement of the circle Bloch line ${ }^{[14,15]}$.

\section{Results and Discussion}

Fig.3a shows an under-focused Lorentz microscopy image of a cross-tie wall in Permalloy obtained with the objective lens completely switched off. The main wall is close to the $y$-axis of the holder and the direction of the magnetization vector $\vec{M}$ is denoted by long black arrows in Fig.3a. The remnant magnetic field is measured using a two step procedure as follows. In the first step, we tilt the holder along $y$-axis (increasing $\beta$ angle), so that an in-plane component of the remnant magnetic field is applied to the specimen in the $x$ direction (Fig.2). Fig.3b-3d show an example for doing such thing, in which the circle Bloch line marked ' $\mathrm{B}$ ' moves along the main wall. In the second step, we keep $\beta$ amount constant, and increase the objective lens current by a very small amount. Fig.4a-4d show the resulting movement of the circle Bloch line ' $\mathrm{B}$ ' along the main wall, but now in the opposite direction compared with that shown in Fig.3, which indicates that the remnant magnetic field is opposite to that of the objective lens field.

The plot in Fig.5a shows the displacement, $\Delta d$, of the circle Bloch line ' $\mathrm{B}$ ' along the main wall as a function of the tilting angle $\beta$. The displacement $\Delta d$ is defined to be positive for a downward movement of circle Bloch line 'B'. A linear relationship is seen. However, the best fit straight line does not pass through the origin, which implies that the specimen plane is not exactly horizontal at $\beta=0$. For $\beta$-tilt angle $1.32^{\circ}$, the displacement $\Delta d$ of the

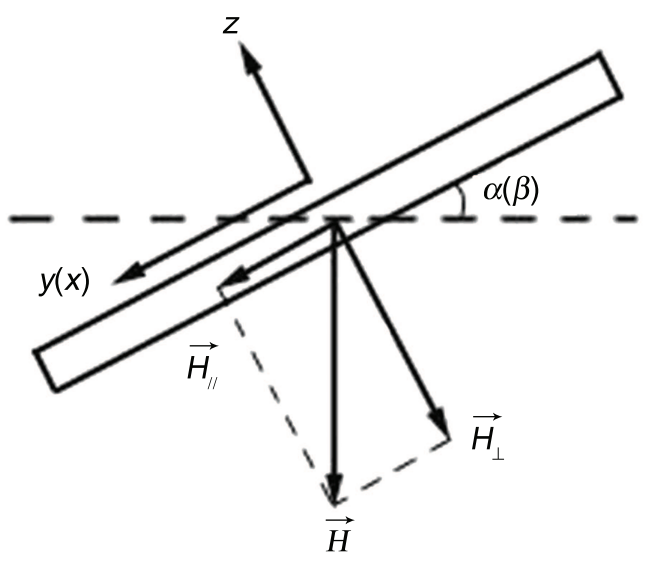

Fig.2 Schematic to show how tilting the holder along the $y(x)$ axis induces an in-plane component of the perpendicular magnetic field in the $x(y)$ direction.

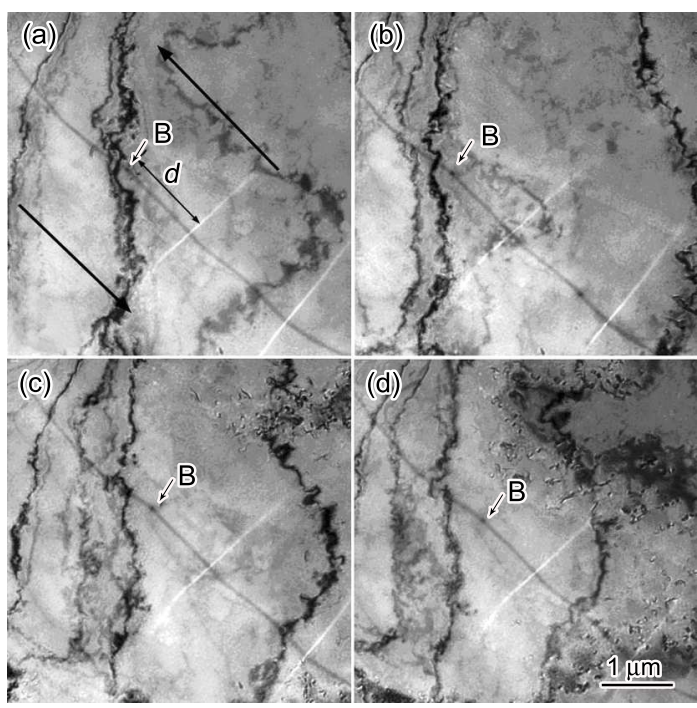

Fig.3 In situ Lorentz microscopy images of cross-tie wall movements due to specimen tilting along $y$ axis: (a) $\beta=0^{\circ}$, (b) $\beta=0.5^{\circ}$, (c) $\beta=1.0^{\circ}$, (d) $\beta=1.32^{\circ}$. 
circle Bloch line B is about $200 \mathrm{~nm}$. Fig.5b shows the plot showing the displacement $\Delta d$ of the circle Bloch line B as a function of objective lens current (expressed as a percentage of the fully saturated value). From this figure we can see that an increasing of the objective lens current of about $0.087 \%$ can move the circle Bloch line ' $\mathrm{B}$ ' back to its origin position, i.e. $\Delta d=0 \mathrm{~nm}$. Assuming the $100 \%$ excited objective lens magnetic field is about $2 \mathrm{~T}$ (20000 Oe), the remnant magnetic field is therefore estimated to be about 17 Oe. Since the remnant magnetic field lies opposite to that of the objective lens magnetic field, it is expected that the rising is not from the objective lens, but from the minilens and/or the Lorentz lens. In practice the remnant magnetic field can be compensated by increasing the objective lens current by a known amount.

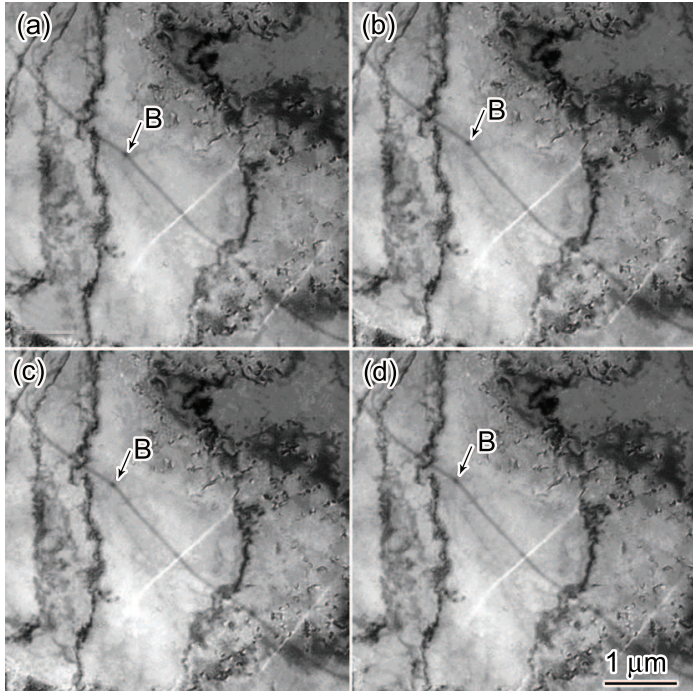

Fig.4 In situ Lorentz microscopy images of cross-tie wall movements due to increasing the current of the objective lens: (a) $0.05 \%$, (b) $0.10 \%$, (c) $0.128 \%$, (d) $0.139 \%$.

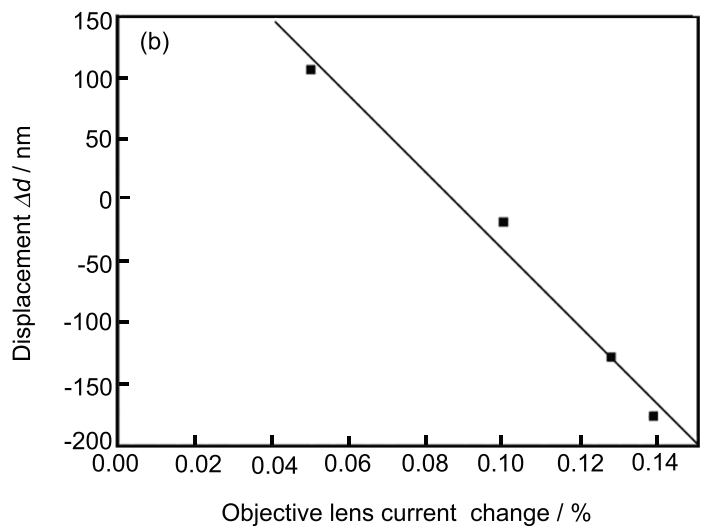

Fig.5 Plots of the displacement of circle Bloch line along the main wall as a function of tilt angle $\beta$ (a) and objective lens current changes (\%) (b).

\section{Conclusions}

From an investigation of a test specimen of Permalloy, we have confirmed the existence of a remnant magnetic field in the Lorentz mode of a FEI Tecnai F20 TEM. By using a novel method of only adjusting sample tilt and the objective lens current, the remnant magnetic field was estimated to be about 17 Oe in a direction opposite to that of the objective lens, and the remnant field can be compensated by increasing the objective lens current in a controlled manner. 
Acknowledgements - This work was supported by National Natural Science Foundation of China (No.10776037).

\section{REFERENCES}

[1] P.B. Hirsh, A. Howie, R.B. Nicholson, D.W. Pashley and M.J. Whelan, Electron Microscopy of Thin Crystals (London: Butterworths, 1965) p.292.

[2] J.N. Chapman and M.R. Scheinfein, J Magn Magn Mater 200 (1999) 729.

[3] K. Saito, H.S. Park, D. Shindo and Y. Yoshizawa, J Magn Magn Mater 305 (2006) 304.

[4] J.N. Chapman, I.R. MacFadyen and S. Mcvitie, IEEE Trans Magn 26 (1990) 1506.

[5] M. Inoue, T. Tomita, M. Naruse, Z. Akase, Y. Murakami and D. Shindo, J Electr Micros 54 (2005) 509.

[6] J.W. Lau, M.A. Schofield and Y. Zhu, Ultramicroscopy 107 (2007) 396.

[7] V.V. Volkov, D.C. Crew, Y. Zhu and L.H. Lewis, Rev Scient Instr 73 (2002) 2298.

[8] Y.R. Cui, H. Liu, Y. Shi, X.M. Li and H. Ma, Foundry Technol 26 (2005) 1145.

[9] J.C. Li, Southern Iron Steel 116 (2000 ) 7.

[10] E.E. Huber, D.O. Smith and J.B. Goodenough, J Appl Phys 29 (1958) 294.

[11] W. Burger, Phys Status Solidi 4 (1971) 713.

[12] W. Burger, Phys Status Solidi 4 (1971) 723.

[13] W. Burger, Phys Status Solidi 13 (1972) 429.

[14] H.Y. Wang, X.F. Dai, Y.G. Wang, X.F. Duan and G.H. Wu, Mater Trans 48 (2007) 2255.

[15] H.H. Liu, X.K. Duan, R.C. Che, Z.F. Wang and X.F. Duan, Mater Lett 62 (2008) 2654. 